\title{
Quantum Theoretical and Computational Controlling for Corral at Matter Surface*
}

\author{
Quan-Fang Wang \\ Mechanical and Automation Engineering \\ The Chinese University of Hong Kong, N.T., Hong Kong \\ E-mails: quanfangwang@yahoo.co.jp;qfwang@mae.cuhk.edu.hk
}

In physics and chemistry realms, the exciting quantum field would play a major role. ${ }^{1-3}$ Particularly, quantum control area should have significant and powerful development tendency. With a numerous attempts in a variety of fields, the extremely frontier area move forward to control elementary particles. Lasting effects must boost the appearing of breakthrough soonerafter. As is well known, a mount of milestone works lie on the controlling of atomic and molecules dynamics. Naturally, future researches will concentrate in quantum controlling at nucleus scale.

How to achieve the controlling of particles at the surface? The objective particles should be regarded as atoms, molecules and nano-particles, etc at matter surface (e.g. crystals, metal, et al). Observing the Scanning Tunneling Microscope (STM) image, rectangular corral (iron on copper (111) citing $^{6}$ ), Figure 1 to represent quantum dot at metal surface.

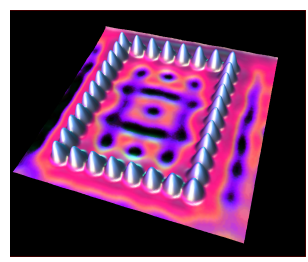

Figure 1. STM image of corral

It would be highlighted to aim at changing the state of particle with the external forcing, such as electromagnetic field, Ti: Sapphire shaped laser/utra-short terahertz $(\mathrm{THz})$ pulse.

Bearing in mind that the purpose of the work is to consider controlling of particle which appeared in quantum corral at matter surface. Deduction of the physical model can be cited in reference ${ }^{5 e}$. Let $\Omega$ be an open bounded set of $\mathbf{R}^{3}$ and $Q=(0, T) \times \Omega$ for $T>0$. Thus, for $\mathbf{x}=\left(x_{1}, x_{2}, x_{3}\right),(\mathbf{x}, t) \in Q$ and positive integral $n_{c}$, many body dynamics has a simultaneous form of

$$
\left\{\begin{array}{l}
i \hbar \sum_{j=1}^{n_{c}} \psi_{t}^{j}+\frac{\hbar^{2}}{2 M} \sum_{j=1}^{n_{c}} \psi_{\mathbf{x x}}^{j}+\sum_{j=1}^{n_{c}} \phi^{j} \psi^{j}+e \sum_{j=1}^{n_{c}} u \psi^{j}=0 \\
\frac{1}{c^{2}} \sum_{j=1}^{n_{c}} \phi_{t t}^{j}-\sum_{j=1}^{n_{c}} \phi_{\mathbf{x x}}^{j}+\left(\frac{m c}{\hbar}\right)^{2} \sum_{j=1}^{n_{c}} \phi^{j} \\
=\sum_{j=1}^{n_{c}}\left|\psi^{j}\right|^{2}+e^{\prime} \sum_{j=1}^{n_{c}} v \phi^{j}
\end{array}\right.
$$

* Related poster "Quantum control for rings/corral at matter surface" refer The 238th ACS National Meeting (fall), Wasington DC, Aug. 16-20, 2009 for $\left.\left(\psi^{j}, \phi^{j}, \phi_{t}^{j}\right)\right|_{t=0}=\left(\psi_{0}^{j}, \phi_{0}^{j}, \phi_{1}^{j}\right)$, where $\psi^{j}$, a complexvalued function, represents probability amplitudes of $j$ th nucleon field, and $\phi^{j}$, a real-valued function, represents probability amplitudes of $j$-th meson field for $j=1,2, \ldots, n_{c}$. Here $e$ and $e^{\prime}$ denote the charges of neutron and meson, respectively. The model (1) represents a many body dynamics under time depended control $u(t)$ and $v(t)$, which corresponding to external ultra short (e.g. femtosecond/attosecond) laser pulses in real lab.

Referring paper ${ }^{4}$ to give a mathematical setting. Denote $\mathbf{u}(t)=(u(t), v(t))$, and quantum optimal control pairing $\mathbf{u}^{*}=\left(u^{*}, v^{*}\right)$ for many body system (1). Introduce two Hilbert spaces $H=L^{2}(\Omega)$ and $V=H_{0}^{1}(\Omega)$ with usual norm and inner products. ${ }^{4}$ Thereby the embeddings in Gelfand triple space $V \hookrightarrow H \hookrightarrow V^{\prime}$ are continuous, dense and compact. Suppose $\mathcal{U}=L^{2}(0, T)$ is the space of laser controls $u$ and $v$. Let $\mathcal{U}_{a d}$ be a closed and convex admissible set of $\mathcal{U}$. The cost criteria associated with (1) is given by

$$
\begin{aligned}
J(\mathbf{u})= & \epsilon_{1}\left(1-\sum_{j=1}^{n_{c}} \int_{\Omega} \psi_{f}^{j}(\mathbf{u}) \psi_{\text {target }}^{j} d \mathbf{x}\right) \\
& +\epsilon_{2}\left(1-\sum_{j=1}^{n_{c}} \int_{\Omega} \phi_{f}^{j}(\mathbf{u}) \phi_{\text {target }}^{j} d \mathbf{x}\right)+(\mathbf{u}, \mathbf{u})_{\mathcal{U}^{2}},
\end{aligned}
$$

for all $\mathbf{u} \in \mathcal{U}_{a d}^{2}$, where $\psi_{\text {target }}^{j}, \phi_{\text {target }}^{j} \in V$ are target states, $\psi_{f}^{j}(\mathbf{u}), \phi_{f}^{j}(\mathbf{u})$ are observed final states, respectively. Here, $\epsilon_{1}$ and $\epsilon_{2}$ are weighted coefficients for balancing the values of inherent and running costs.

In order to find the quantum optimal $\mathbf{u}^{*}$, numerous algorithms have been developed for quantum control. ${ }^{3}$ In this work, Wang semi-discrete algorithm ${ }^{5}$ (space variable $\mathbf{x}$ discrete and time $t$ continuous) is executed in controlling quantum dynamics for meeting the needs of wave-particle duality. More precisely, finite element method is used in numerical approach based on the variational method in Hilbert space, the modified nonlinear conjugated gradient method is utilized in optimality minimization. ${ }^{5}$

Because the particles are lying on the matter surface, it's appropriate to consider the two-dimensional case for spatial variable. Set $m=\hbar=c=1$ for simplicity. Let $\Omega=(0,50) \times(0,50)$, start time $t_{0}=0.0(\mathrm{fs})$, iteration step $n \leq 5$, time step size $d t=0.5$, then $T=5 d t$. Define two appendix functions for initial states configuration.

$$
\begin{aligned}
\Psi\left(x^{\prime}, t, v_{1}\right)= & \frac{3 \sqrt{2}}{4 \sqrt{1-v_{1}^{2}}} \sec ^{2} \frac{1}{2 \sqrt{1-v_{1}^{2}}}\left(x^{\prime}-v_{1} t-x_{0}\right) \\
& \times \exp \left(i\left(v_{1} x^{\prime}+\frac{\left(1-v_{1}^{2}+v_{1}^{4}\right)}{2\left(1-v_{1}^{2}\right)} t\right)\right)
\end{aligned}
$$




$$
\Phi\left(x^{\prime}, t, v_{2}\right)=\frac{3}{4\left(1-v_{2}^{2}\right)} \sec ^{2} \frac{1}{2\left(1-v_{2}^{2}\right)}\left(x^{\prime}-v_{2} t-x_{0}\right),
$$

where $x^{\prime}$ is space variable, $v_{1}$ and $v_{2}$ are velocities of nucleon and meson, respectively. Set wave propagation with $v_{1}=5 / 6, v_{2}=-5 / 6$. Setting location cent $\epsilon$ $x_{0}=25.0$, two particles locations $y_{10}=15.0, y_{20}=35$. ( Gaussian enveloped function $s(n, t)=\exp (-6 \pi(t-(n$ $\left.\frac{1}{2} d t\right)^{2}$ ), The initial control wave functions are given by

$$
\begin{aligned}
& u^{n}(t)=s(n, t)\left(\sin \left(300 t+\frac{\pi}{6}\right)+\sin \left(100 t+\frac{\pi}{3}\right)\right), \\
& v^{n}(t)=s(n, t)\left(\sin \left(240 t+\frac{\pi}{3}\right)+\sin \left(450 t+\frac{\pi}{6}\right)\right) .
\end{aligned}
$$

Let $x_{0}=20.0, p_{10}=-15.0, p_{20}=25.0, p_{1}=x_{0}+p_{10}+$ $v_{1}(n-1) d t, p_{2}=x_{0}+p_{20}+v_{2}(n-1) d t$. Then initial ground states given by

$$
\begin{aligned}
\psi_{0}= & \sum_{j=1}^{9} \Psi\left(0, \sqrt{\left(x_{1}-5 j\right)^{2}+\left(x_{2}-p_{1}\right)^{2}}, v_{2}\right) \\
& +\sum_{j=1}^{9} \Psi\left(0, \sqrt{\left(x_{1}-5 j\right)^{2}+\left(x_{2}-p_{2}\right)^{2}}, v_{2}\right) \\
& +\sum_{j=1}^{4} \Psi\left(0, \sqrt{\left(x_{1}-p_{1}\right)^{2}+\left(x_{2}-10 j+5\right)^{2}}, v_{2}\right) \\
& +\sum_{j=1}^{4} \Psi\left(0, \sqrt{\left(x_{1}-p_{2}\right)^{2}+\left(x_{2}-10 j+5\right)^{2}}, v_{2}\right)
\end{aligned}
$$

and $\phi_{0}$ is the same structure instead of $\Psi$ with $\Phi$ and $v_{2}$ with $v_{1}$. The states transition of nucleons see Figure 2 .
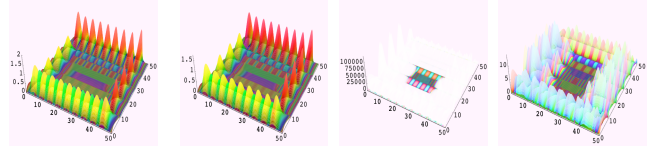

Figure 2. $\psi(t)$ iteration

Their contour plots refer to Figure 3.
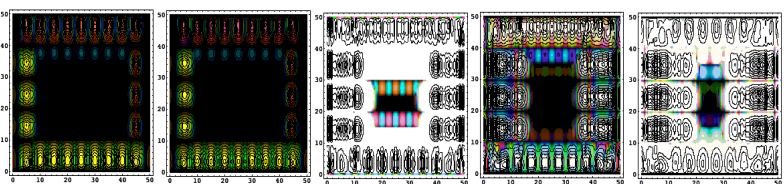

Figure 3. Contour plots of $\psi(t)$.

The meson states transferred in Figure 4.
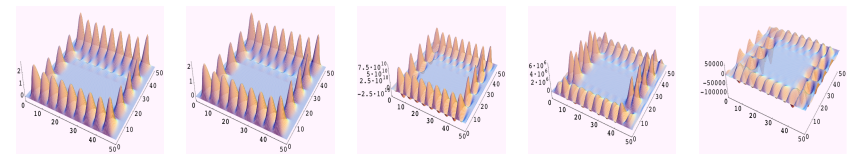

Figure 4. $\phi(t)$ iteration

The contour plots can be found in Figure 5 .
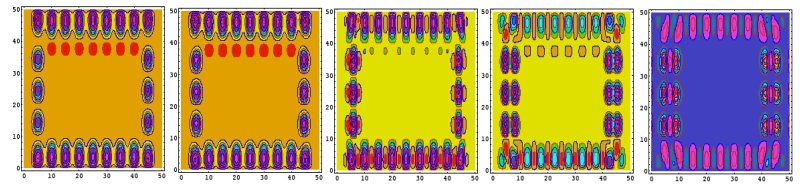

Figure 5. Contour plots of $\phi(t)$.

Then by limited iteration steps, the optimal control variables is obtained as

$$
\begin{aligned}
& u^{*}(t)=-0.00226639+\sin \left(\frac{\pi}{6}+300 t\right)+\sin \left(\frac{1}{3}(\pi+300 t)\right), \\
& v^{*}(t)=0.00129947+\sin \left(\frac{\pi}{3}+250 t\right)+\sin \left(\frac{\pi}{6}+450\right) .
\end{aligned}
$$

Their graphics are shown in Figure 6. The objective functions iteration and their error values are shown in Figures 7 for comparing at each step.
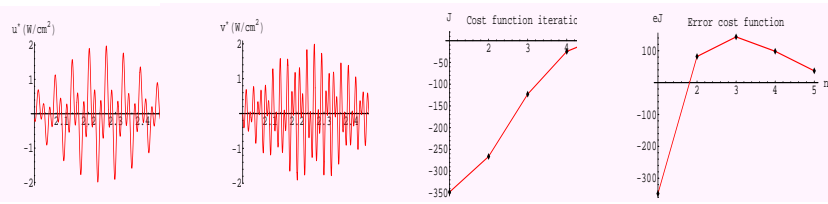

Figure 6. $u^{*}(t), v^{*}(t)$,

Figure 7. $J(\mathbf{u})$, eJ $(\mathbf{u})$.

Finally, the calculated optimality value $J\left(\mathbf{u}^{*}\right)=12.2588$ and minimization cost error $e J\left(\mathbf{u}^{*}\right)=36.9362$. The total computer running time is 10831.3 second, and maximum used memory is 105435368 bits.

This work regarded quantum corral at matter surface as the objective motions to perform controlling. The computational result is evident the effectiveness and feasibility of theoretic predication.

It's benefit to explore the theoretical controlling for guiding the real experiment executives. ${ }^{5}$ The realization of the real control at surface will be desired at the advance experiments technology with optical tools.

Acknowledgement The author express full gratitude to 238th ACS National Meeting (Fall) and Dr. F. Tao (LBL, University of California at Berkeley) for previous work. ${ }^{5 f}$ The author faithfully thank to Prof. Y. Ohtsuki (Department of chemistry, Tohoku University, Japan) for helpful comments to improve the manuscript.

\section{References}

(1) Schrödinger, E. Nature 1952, 169, 538-542.

(2) Yukawa, H. Proceeding of Japan Physics and Mathematics Society 1934, 17, 48-56.

(3) (a) Rice, S. A.; Zhao, M. Optical control of molecular dynamics 2000, New York, John Wiley. (b) Warren, W.; Rabitz, H.; Dahleh, M. Science 1993, 259, 1581-1585.

(4) Lions, J. L. Optimal Control of Systems Governed by Partial Differential Equations 1971, BerlinHeidelberg-New York, Springer-Verlag.

(5) (a) Wang, Q. F. Proceeding of American Control Conference 2006, 1032-1037. (b) Wang, Q. F.; Rabitz, H. Gordon Research Conference 'Quantum Control of Light and Matter', USA 2007, Poster. (c) Wang, Q. F. Applied Mathematics and Computation 206, 2008, 276-289. (d) Wang, Q.F. IET Control Theory \& Applications 3(9), 2009, 11751182. (e) Wang, Q. F.; Cao, C. Proceeding of the European Control Conference, 2009, 168-172. (f) Wang, Q. F. 238th ACS National Meeting (fall) 2009, Poster.

(6). http://www.almaden.ibm.com/vis/stm/corral.html 